International Journal of Pure and Applied Mathematics

Volume 111 No. 2 2016, 331-342

ISSN: 1311-8080 (printed version); ISSN: 1314-3395 (on-line version)

url: http://www.ijpam.eu

doi: 10.12732/ijpam.v111i2.16

\title{
MODELING THE UNSTEADY CONTACT OF SPHERICAL SHELL MADE WITH APPLYING THE ADDITIVE TECHNOLOGIES WITH THE PERFECTLY RIGID STAMP
}

\author{
Gregory V. Fedotenkov ${ }^{1}$, Elena Yu. Mikhailova ${ }^{2}$, \\ Elena L. Kuznetsova ${ }^{3}$, Lev N. Rabinskiy ${ }^{4}$ \\ 1,2,3,4 Moscow Aviation Institute \\ National Research University \\ 125993, Volokolamsk Highway 4, Moscow, RUSSIAN FEDERATION
}

\begin{abstract}
Modeling the unsteady contact of thin spherical shell with the rigid obstacle was carried out. Problem was solved on base of numerical-analytical method with usage of integral representation for the shell points' normal displacements through the influence functions. There was conducted the comparison of modeling results with those of the experimental investigations for polymeric shells made with the layer-by-layer laser synthesis. Influence of the synthesized shell geometry quality on the process of its unsteady deformation under the load was studied.
\end{abstract}

AMS Subject Classification: 97M10, 74K25

Key Words: modeling, unsteady contact, spherical shell, compression test, layer-by-layer laser synthesis, geometry defect

\section{Problem Definition}

There is considered the process of the steady contact interaction of the thin elastic spherical shell of $R$ radius and $h$ thickness with two perfectly rigid stamps one of which is the support stationary stamp and the second stamp moves with some specified velocity $2 \mathrm{~V}$.

At the initial time point the shell one pole supports on the stationary stamp and the mobile stamp begins to press on the second pole. The both poles' sur-

Received: October 15, 2016

Revised: October 29, 2016

Published: December 11, 2016

$\S_{\text {Correspondence author }}$ (c) 2016 Academic Publications, Ltd.

url: www.acadpubl.eu 
faces during the entire deformation process remain in parallel to each other that leads to axial-symmetric problem definition. Taking additionally into consideration the problem symmetry relatively to horizontal plane $\prod$ which is in parallel to the stamps' surfaces and divides the spherical shell in halves, the problem boils down to the equivalent axial-symmetric problem of the unsteady contact interaction with the mobile stamps the velocity of each of them is $V$ (Figure 1).
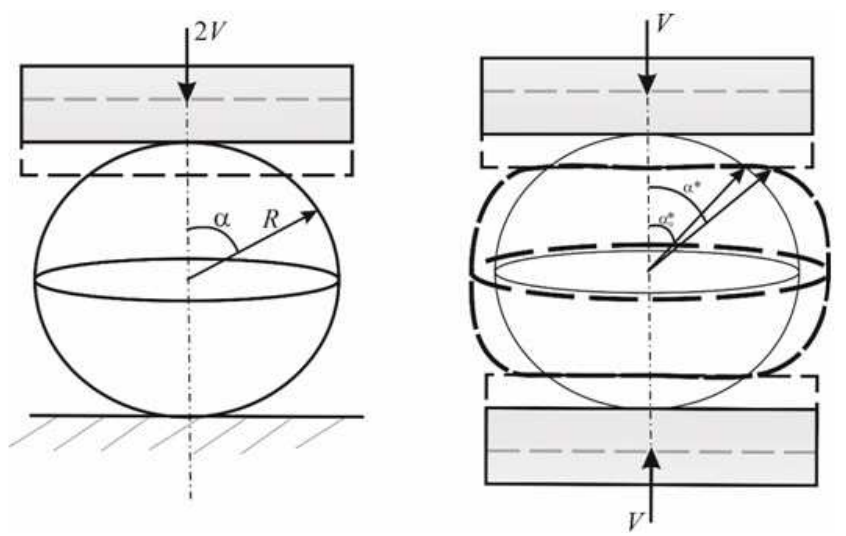

Figure 1:Problem definition.

For theoretical description of the shell deformation process there are used Timoshenko model movement equations [1] and [2] written in the spherical coordinate system with center on the shell axis and angle $\alpha \in[0, \pi]$ (dots above the characters hereinafter mean the time derivatives):

$$
\begin{gathered}
\ddot{\mathbf{w}}=\mathbf{L w}+\mathbf{p}, \mathbf{w}=(u, w, \chi)^{T}, \\
\mathbf{L}=\left(L_{i j}\right)_{3 \times 3}, \mathbf{p}=(0, p, 0)^{T}, \\
L_{11}=l_{2}-\frac{1}{\sin ^{2} \alpha}+\eta^{2}\left(2-k^{2}\right), L_{12}=\left[2\left(1-\eta^{2}\right)+\eta^{2} k^{2}\right] \frac{\partial}{\partial \alpha} \\
L_{13}=-\gamma^{2} l_{2}+\eta^{2} k^{2}, L_{21}=-\left[2\left(\eta^{2}-1\right)+\eta^{2} k^{2}\right] l_{1}, \\
L_{22}=\eta^{2} k^{2} l_{2}-4\left(1-\eta^{2}\right), L_{23}=\eta^{2} k^{2} l_{1}, L_{31}=\gamma^{-2} L_{13}, \\
L_{32}=-\gamma^{-2} \eta^{2} k^{2} \frac{\partial}{\partial \alpha}, L_{33}=-L_{31}, \\
l_{1}=\frac{\partial}{\partial \alpha}+\operatorname{ctg} \alpha, l_{2}=\frac{\partial^{2}}{\partial \alpha^{2}}+\frac{\partial}{\partial \alpha} \operatorname{ctg} \alpha, k^{2}=\frac{5}{6},
\end{gathered}
$$


where $u, w$ - shell tangential and normal displacements; $\chi$ - turn angle of normal to median surface before the fiber deformation due to shear deformation, $p$ - contact pressure.

All quantities and parameters are reduced to dimensionless form (dimensional parameters are marked with dash)

$$
\begin{gathered}
u=\frac{u^{\prime}}{R}, w=\frac{w^{\prime}}{R}, \tau=\frac{c_{1} t}{R}, \gamma^{2}=\frac{h^{2}}{12 R^{2}}, \eta^{2}=\frac{c_{2}^{2}}{c_{1}^{2}}, \\
p=\frac{p^{\prime}}{\sigma}, \sigma=2 \sqrt{3}(\lambda+2 \mu) \gamma, \\
c_{1}^{2}=\frac{\lambda+2 \mu}{\rho}, c_{2}^{2}=\frac{\mu}{\rho}, k_{1}^{2}=\eta^{2}\left(2-k^{2}\right), k_{2}^{2}=\eta^{2}\left(k^{2}-2 \gamma^{2}\right), \\
k_{3}^{2}=\eta^{2}\left(k^{2} \gamma^{-2}-2\right), a^{2}=4\left(1-\eta^{2}\right),
\end{gathered}
$$

where $t$-dimensional time; $c_{1}, c_{2}$-dimensional velocities of extension-compression and shift waves in the shell material; $\rho$ - dimensional density of the shell material; $\lambda, \mu$ - shell material Lame elastic constants.

Contact area boundaries in the vicinity of the poles are changing during interaction. It is assumed that the contact between the shell and the stamps occurs under the free slipping conditions. In such case the following relationships between the stamp displacements, the shell normal displacements and contact pressure take place

$$
\begin{gathered}
w(\alpha, \tau)=1-\cos \alpha-V \tau, \alpha \in\left[0, \alpha^{*}\right] \cup\left[\pi-\alpha^{*}, \pi\right] \\
p(\alpha, \tau)<0, \alpha \in\left[0, \alpha^{*}\right] \cup\left[\pi-\alpha^{*}, \pi\right] \\
p(\alpha, \tau)=0, \alpha \notin\left[0, \alpha^{*}\right] \cup\left[\pi-\alpha^{*}, \pi\right]
\end{gathered}
$$

Angular dimension of the contact areas $\alpha^{*}$ depends on the stamp movement velocity and the shell surface deformation. In the zero approach it is defined from the intersection condition of the stamps' surfaces with the shell non-deformed surface.

$$
\cos \alpha_{0}^{*} 1-V \tau \text {. }
$$

Taking into consideration the shell deformation the angular dimension is defined from the condition

$$
w(\alpha, \tau)+\cos \alpha+V \tau-1=\left\{\begin{array}{r}
0, \alpha \in\left[0, \alpha^{*}\right] \cup\left[\pi-\alpha^{*}, \pi\right] \\
>0, \alpha \in\left[0, \alpha^{*}\right] \cup\left[\pi-\alpha^{*}, \pi\right]
\end{array}\right.
$$

Initial conditions are zero:

$$
\left.u\right|_{\tau=0}=\left.w\right|_{\tau=0}=\left.\chi\right|_{\tau=0}=\left.\dot{u}\right|_{\tau=0}=\left.\dot{w}\right|_{\tau=0}=\left.\dot{\chi}\right|_{\tau=0}=0 .
$$




\section{Solution Method and Algorithm}

Based on the superposition principle $[1,2,3,4]$ the shell normal displacements are connected with the contact pressure by the integral relationship

$$
\begin{gathered}
w(\alpha, \tau)=2 \pi \int_{0}^{\tau} \int_{0}^{\alpha^{*}(t)} G_{2}(\alpha, \vartheta, \tau-t) p(\vartheta, t) \sin \vartheta d \vartheta d t+ \\
+2 \pi \int_{0}^{\tau} \int_{\pi-\alpha^{*}(t)}^{\pi} G_{2}(\alpha, \vartheta, \tau-t) p(\vartheta, t) \sin \vartheta d \vartheta d t .
\end{gathered}
$$

where $G_{2}(\alpha, \vartheta, \tau)$ - influence functions for spherical shell which represents the normal displacements $w=G_{2}(\alpha, \vartheta, \tau)$ as the solution of problem (1), (5) at $p=p_{0}=\delta(\tau) \delta(\alpha-\vartheta)$, where $\delta(\tau)$ - Dirac delta-function. In this case let's introduce the following notations for the shell tangential displacements and the normal turn angle to median surface: $u=G_{1}(\alpha, \vartheta, \tau), \chi=G_{3}(\alpha, \vartheta, \tau)$.

To construct the influence function $G_{2}(\alpha, \vartheta, \tau)$ we'll apply the variables separation. Let's represent the defined and required functions in the form of Fourier series per Legendre polynomials $P_{n}(x)$ and Gegenbauer polynomials $C_{n-1}^{3 / 2}(x)=d P_{n}(x) / d x$ :

$$
\begin{gathered}
{\left[\begin{array}{l}
G_{1} \\
G_{3}
\end{array}\right]=-\sin \alpha \sum_{n=1}^{\infty}\left[\begin{array}{l}
G_{1 n}(\vartheta, \tau) \\
G_{3 n}(\vartheta, \tau)
\end{array}\right] C_{n-1}^{3 / 2}(\cos \alpha),} \\
{\left[\begin{array}{l}
G_{2} \\
p_{0}
\end{array}\right]=\sum_{n=0}^{\infty}\left[\begin{array}{l}
G_{2 n}(\vartheta, \tau) \\
p_{0 n}(\vartheta, \tau)
\end{array}\right] P_{n}(\cos \alpha), p_{0 n}=\frac{2 n+1}{2} P_{n}(\cos \vartheta) \delta(\tau) .}
\end{gathered}
$$

After introduction (7) into (1) and application of Laplace time transformation to obtained equations we get the algebraic equations set with respect to images $G_{1 n}^{L}(\vartheta, s), G_{2 n}^{L}(\vartheta, s), G_{3 n}^{L}(\vartheta, s)$ (superscript " $L$ " for function means Laplace image, $s$-transformation parameter) with solution for $G_{2 n}^{L}$ :

$$
\begin{gathered}
G_{2 n}^{L}=\frac{2 n+1}{2} \frac{\sum_{j=0}^{2} S_{j}\left(s^{2}, n_{1}^{2}\right)}{\sum_{j=0}^{3} T_{j}\left(s^{2}, n_{1}^{2}\right)} P_{n}(\cos \vartheta), \\
S_{0}\left(s, n_{1}\right)=k_{3}^{2}\left(k_{1}^{2}+k_{2}^{2}\right),
\end{gathered}
$$




$$
\begin{gathered}
S_{1}=\left(s, n_{1}\right)=s\left(k_{1}^{2}-k_{3}^{2}\right)+n_{1}\left[\left(\gamma^{2}-1\right) k_{3}^{2}+k_{1}^{2}+k_{2}^{2}\right], \\
S_{2}\left(s, n_{1}\right)=-\left[s+n_{1}(1+\gamma)\right]\left[s+n_{1}(1-\gamma)\right], T_{0}\left(s, n_{1}\right)=a^{2} S_{0}\left(s, n_{1}\right), \\
T_{1}\left(s, n_{1}\right)=s\left[k_{1}^{2}\left(k_{3}^{2}+a^{2}\right)+k_{3}^{2}\left(k_{2}^{2}-a^{2}\right)\right]+ \\
+n_{1}\left\{k_{3}^{2} k_{1}^{4}+\left[\left(k_{2}^{2}-4+\eta^{2} k^{2}\right) k_{3}^{2}+a^{2}-2 \eta^{2} k^{2}+2 k_{2}^{2}\right] k_{1}^{2}+\right. \\
\left.+\left[\left(\eta^{2} k^{2}-2\right) k_{2}^{2}+\left(\gamma^{2}-1\right) a^{2}-2 \eta^{2} k^{2}+4\right] k_{3}^{2}+\left(a^{2}-4\right) k_{2}^{2}\right\}, \\
T_{2}\left(s, n_{1}\right)=s^{2}\left(k_{1}^{2}-k_{3}^{2}-a^{2}\right)+ \\
+s n_{1}\left[k_{1}^{4}+\left(\eta^{2} k^{2}-3\right) k_{1}^{2}+\left(\gamma^{2}-1\right) k_{3}^{2}-2\left(a^{2}-\eta^{2} k^{2}\right)+k^{2}+4\right]+ \\
+n_{1}^{2}\left\{k_{1}^{4}+\left[\gamma^{2}\left(2+k_{3}^{2}\right)+2\left(\eta^{2} k^{2}-2\right)\right] k_{1}^{2}+\right. \\
\left.+\gamma^{2} k_{3}^{2}\left(\eta^{2} k^{2}-2\right)+\eta^{2} k^{2} k_{2}^{2}+\left(\gamma^{2}-1\right)\left(a^{2}-4\right)\right\}, \\
T_{3}\left(s, n_{1}\right)=S_{2}\left(s, n_{1}\right)\left(s+\eta^{2} k^{2} n_{1},\right.
\end{gathered}
$$

where $n_{1}^{2}=n(n+1)$.

Note that $G_{21 n}^{L}$ image's structure represents the proper rational fraction, and therefore at the problem defined parameters its original is determined in analytical way using the second expansion theorem for Laplace transformation. Thus, the influence is defined by $G_{2}(\alpha, \vartheta, \tau)$ expansion the coefficients of which are the originals of expressions (8) and determined analytically. Coefficient originals have the structure

$$
G_{2 n}(\vartheta, \tau)=\frac{2 n+1}{2} P_{n}(\cos \vartheta) \sum_{k=1}^{3} A_{n k} \sin \left(\omega_{n k} \tau\right)
$$

where $A_{n k}, \omega_{n k}$ - real constants which depend on the shell geometrical parameters and the material elastic constants.

Let's reduce the originals of the first five coefficients $G_{2 n}(\vartheta, \tau), n=\overline{0,4}$ for the shell with radius of $R=80 \mathrm{~mm}$ and thickness of $h=2 \mathrm{~mm}$ for polyamide with density of $1.13 \mathrm{~g} / \mathrm{cm}^{3}$, Young's modulus of $1.735 \mathrm{GPa}$ and Poisson's ratio of 0.43 :

$$
\begin{gathered}
G_{20}(\vartheta, \tau)=G_{20}(\tau)=\frac{\sin a \tau}{2 a} \\
G_{2 n}(\vartheta, \tau)=\frac{2 n+1}{2} P_{n}(\cos \vartheta) \sum_{k=1}^{3} A_{n k} \sin \left(\omega_{n k} \tau\right), n=\overline{1,3}
\end{gathered}
$$




$$
A=\left[\begin{array}{ccc}
1.34 & 0.18 & 1.8 \cdot 10^{-4} \\
0.91 & 0.01 & 10^{-3} \\
0.35 & 10^{-3} & 10^{-3}
\end{array}\right], \omega=\left[\begin{array}{ccc}
0.37 & 2.6 & 13.7 \\
0.1 & 6.2 & 14.9 \\
2.6 & 12 & 18.4
\end{array}\right]
$$

Let's make the variable change $\vartheta=\vartheta-\pi$ in the second integral $I$ (6). Then, taking into account the symmetry of problem $(p(\vartheta+\pi, t)=p(\vartheta, t))$ the integral presentation (6) will have the following form:

$$
\begin{gathered}
w(\alpha, \tau)=2 \pi \int_{0}^{\tau} \int_{0}^{\alpha^{*}(t)} \tilde{G}_{2}(\alpha, \vartheta, \tau-t) p(\vartheta, t) \sin \vartheta d \vartheta d t \\
\tilde{G}_{2}(\alpha, \vartheta, \tau-t)=G_{2}(\alpha, \vartheta, \tau-t)+G_{2}(\alpha, \vartheta+\pi, \tau-t) .
\end{gathered}
$$

Using this presentation, the boundary conditions (2) and expressions (3), (4), we'll get the resolving equations set relative to contact pressure

$$
\begin{aligned}
2 \pi \int_{0}^{\tau} \int_{0}^{\alpha^{*}(t)} \tilde{G}_{2}(\alpha, \vartheta, \tau-t) p(\vartheta, t) \sin \vartheta d \vartheta d t & =1-\cos \alpha-V \tau, \cos \alpha_{0}^{*}=1-V \tau \\
& =1 \\
w(\alpha, \tau)+\cos \alpha+V \tau-1 & =\left\{\begin{array}{cc}
0, & \alpha \in\left[0, \alpha^{*}\right] \\
>0, & \alpha \notin\left[0, \alpha^{*}\right]
\end{array}\right.
\end{aligned}
$$

To solve set (10), let's use the numeric-analytical algorithm with the contact area angular dimension correction iterative procedure based on the mechanical quadrature method. Grid with uniform pitch $\Delta$ along the angular coordinate and time is applied to space-time domain $R_{t \vartheta}^{2}$ (Figure 2):

$$
\begin{gathered}
t_{i}=i \Delta, \xi_{j}=j \Delta, R_{t \xi}^{2}=\bigcup_{i} K_{j} K_{i j}(i=0,1,2, \ldots, j \in Z), \\
K_{i j}=\left\{(t, \vartheta) \mid t_{i-1} \leq t \leq t_{i}, \vartheta_{j-1} \leq \vartheta \leq \vartheta_{j}\right\} .
\end{gathered}
$$




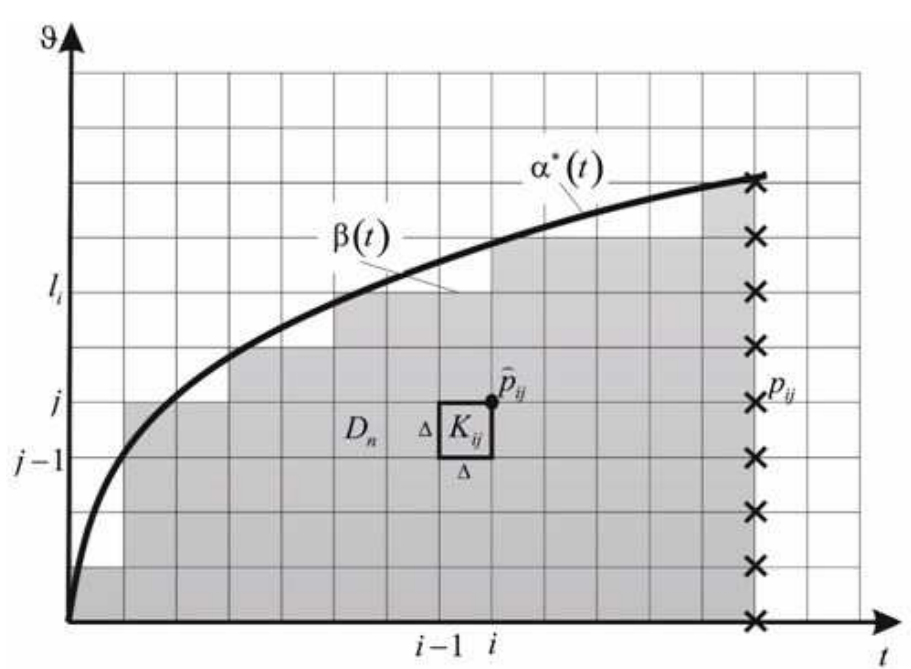

Figure 2: Space-time domain of integration.

Let's place the grid functions in correspondence with functions of one and two variables $w(\vartheta, t), p(\vartheta, t), G_{2}(\alpha, \vartheta, t), \alpha_{0}^{*}(t), \alpha^{*}(t)$ :

$$
\begin{gathered}
w_{m n}=w\left(\vartheta_{m}, t_{n}\right), p_{m n}=p\left(\vartheta_{m}, t_{n}\right), \\
G_{k m n}=G\left(\alpha_{k}, \vartheta_{m}, t_{n}\right), \alpha_{0 n}^{*}=\alpha_{0}^{*}(t), \alpha_{n}^{*}=\alpha^{*}(t) .
\end{gathered}
$$

Boundary of domain $\partial D(\tau)=\left\{\vartheta=\alpha^{*}(t), t \in[0, \tau]\right\}$ is approximately substituted with the piecewise-constant function $\beta(t)$ determined as:

$$
\beta(t)=\Delta\left[\alpha_{0}^{*}(t) / h\right],
$$

where $[\cdot]$ - integral part of a number.

In this case the space-time domain of integration $D_{n}=\{(t, \vartheta): 0 \leq t \leq$ $\left.t_{n}, \quad 0 \leq \vartheta \leq \alpha^{*}(t)\right\}$ at time point $\tau=t_{n}=n h$ is approximated with convex polygon $B_{n}$ consisting of the elementary vertical bars combination:

$$
B_{n}=\bigcup_{i=1}^{n} \bigcup_{j=1}^{l_{i-1}} K_{i j}, l_{i}=\frac{\beta_{i}}{\Delta} .
$$

Then the set (10) discrete analog has the form

$$
\begin{gathered}
\cos \alpha_{0 n}^{*}=1-V \tau_{n}, \\
2 \pi \sum_{i=1}^{n-1} \sum_{j=1}^{l_{i}} a_{k i j} \hat{p}_{i j}+\sum_{j=1}^{l_{n}} a_{k n j} p_{i j}=1-\cos \alpha_{k}, k=0,1,2, \ldots, l_{n-1},
\end{gathered}
$$




$$
\begin{gathered}
w_{m n}=2 \pi \sum_{i=1}^{n} \sum_{j=0}^{l_{i-1}} a_{k i j} \hat{p}_{i j}, \\
w_{m n}+\cos \alpha_{n}+V \tau_{n}-1=\left\{\begin{array}{rr}
0, & \alpha_{n} \in\left[0, \alpha_{n}^{*}\right] ; \\
>0, & \alpha_{n} \in\left[0, \alpha_{n}^{*}\right],
\end{array}\right.
\end{gathered}
$$

where $\hat{p}_{i j}$ - known at the current time pitch with number $n$ grid values of contact pressure, $p_{i j}$ - required at current time pitch with grid values of contact pressure.

Coefficients $a_{k i j}$ of the quadrature formula in (10) are determined as

$$
a_{k i j} \iint_{K_{i j}} \tilde{G}_{2}\left(\alpha_{k}, \vartheta, n \Delta-t\right) d \vartheta d t=\int_{(i-1) \Delta}^{i \Delta} \int_{(j-1) \Delta}^{j \Delta} \tilde{G}_{2}\left(\alpha_{k}, \vartheta, n \Delta-t\right) d \vartheta d t .
$$

Influence function $G_{2}(\alpha, \vartheta, \tau)$ structure allows calculating the values of these coefficients in analytical way. For this purpose, using the first $N+1$ expansion terms (7) and taking into consideration (9), we get

$$
\begin{aligned}
G_{2}(\alpha, \vartheta, \tau) \approx G_{2 N}(\alpha, \vartheta, \tau)= \\
=\frac{\sin a \tau}{2 a}+\sum_{l=1}^{N} \frac{2 n+1}{2} \sum_{q=1}^{3} \sin \left(\omega_{n k} \tau\right) P_{n}(\cos \vartheta) P_{n}(\cos \alpha)
\end{aligned}
$$

Let's determine the antiderivative of function (15):

$$
\begin{gathered}
F_{2 N}(\alpha, \vartheta, \tau)=\iint G_{2 N}(\alpha, \vartheta, \tau) d \vartheta d t= \\
=\frac{\cos a \tau}{2 a^{2}} \vartheta-\sum_{l=1}^{N} \frac{2 n+1}{2} \sum_{q=1}^{3} A_{n k} \frac{\cos \left(\omega_{n k} \tau\right)}{\omega_{n k}} \hat{P}_{n}(\vartheta) P_{n}(\cos \alpha) \\
\hat{P}_{n}(\vartheta)=\int P_{n}(\cos \vartheta) d \vartheta
\end{gathered}
$$

Then

$$
\begin{aligned}
a_{k i j}=\sum_{k, l=0}^{1}(-1)^{k+l}\left\{F_{2 N}[\alpha,(j-k) \Delta\right. & ,(n-i+l) \Delta]+ \\
& \left.+F_{2 N}[\alpha,(j-k+\pi) \Delta,(n-i+l) \Delta]\right\} .
\end{aligned}
$$


Contact pressure calculation algorithm includes the following actions. At each time pitch with number $n$ there is determined the contact area $\alpha_{0 n}^{*}$ angular dimension value in the zero approach using formula (11). Then there are determined the required values of the contact pressure in the grid nodes using the equations set (12). Then the normal displacement $w_{m n}$ grid values are determined using formula (13). Using these values and (13) the contact area $\alpha_{n}^{*}$ angular dimension is corrected. Then there are determined the contact pressure and normal displacement corrected values using the equation set (12) and formula (13). Resultant contact pressure is determined as follows:

$$
\begin{aligned}
R(\tau) & =2 \pi \int_{0}^{\alpha^{*}(\tau)} p(\alpha, \tau) \sin \alpha d \alpha \approx R_{n}=2 \pi \sum_{k=1}^{l_{n}} b_{k} p_{k n} \\
b_{k} & =\int_{(k-1) \Delta}^{k \Delta} \sin \alpha d \alpha=\cos [(k-1) \Delta]-\cos (k \Delta) .
\end{aligned}
$$

\section{Experimental Data}

Process of the shell unsteady contact with the rigid obstacle was studied in experimental way for the spherical thin shells made of polyamide PA2200. Diameter of these shells was equal to $80 \mathrm{~mm}$, wall thickness - $2 \mathrm{~mm}$. There were investigated three variants of shells made by the layer-by-layer laser synthesis on EOS P395S installation. The first variant (Fig. 3a) had the "perfect" geometry and was made using the slicing pitch (thickness of one layer at laser synthesis) of $120 \mu \mathrm{m}$. While preparing the shell second variant there was studied the possibility to increase the slicing pitch to $200 \mu \mathrm{m}$. In this case the shell surface quality deteriorated (Fig. 3b), and the investigation task lied in determination of the model geometry quality deterioration influence degree on mechanical behavior of the finished product under the load. In the third variant there was simulated the geometry construction error when the shell is nor the solid material but represents two surfaces separated with the green powder layer. Such errors of geometry construction appear sometimes in the real structure during its 3D-printing (at model slicing, model geometry format transformation, etc.). Externally such products, particularly the finished spherical shell, do not differ from the high-quality product (Fig. 3a), however their mechanical properties are very poor. 

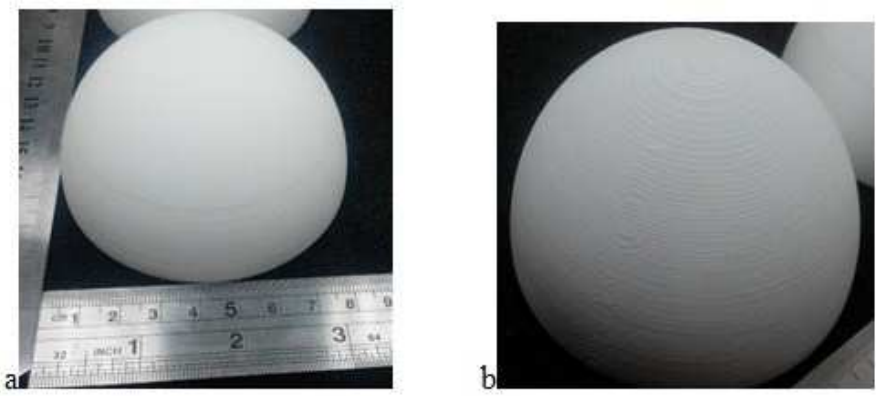

Figure 3:Finished spherical shells with the "perfect" geometry (a) and with the slicing increased pitch $(3 \mathrm{~b})$
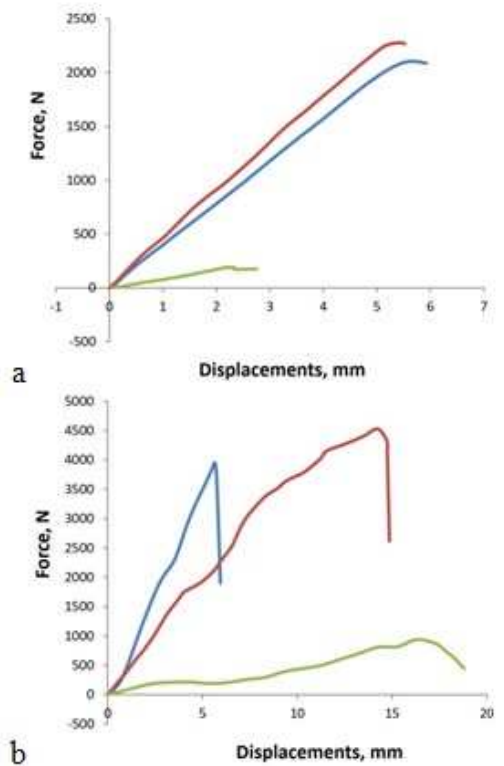

Figure 4: Force-displacement diagram at compression test of the thin-wall spherical shells with the "perfect" geometry (blue) and with artificial deterioration of geometry: slicing pitch increases (red) and with green powder (green). Test at velocity of $1 \mathrm{~mm} / \mathrm{min}$ (a) and $600 \mathrm{~mm} / \mathrm{min}$ (b).

For making the shells there were conducted the compression tests on Instron 5965 installation. Tests were carried out at velocity of $1 \mathrm{~mm} / \mathrm{min}$ and 600 $\mathrm{mm} / \mathrm{min}$. Stability loss critical load was evaluated at low velocities. Then the shells were subject to high-velocity loading up to destruction. Test results are shown in Fig. 4. One can see that the model slicing change leads to slight change of the force-displacement curve. The shell does not lose the bearing 
capacity. Thus, such type of defect cannot be considered as the critical one if there are no requirements to the product surface high quality. Diagrams for the shell consisting of two surfaces separated with green powder lie considerably lower than the first two curves. Rigidity and the stability loss load significantly decreases. Actually the material becomes off-grade. Characteristic appearance of such shells is shown in Fig. 5. Note that the shell with the green powder is not the subject to brittle rupture at the unsteady loading but is collapsed (Figure 5c).

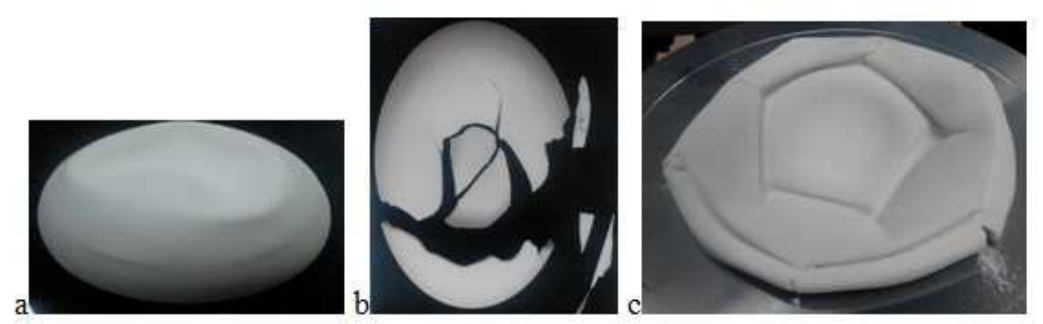

Figure 5: Shells after stability loss at compression test. a - quasi-static test, b, c - unsteady contact

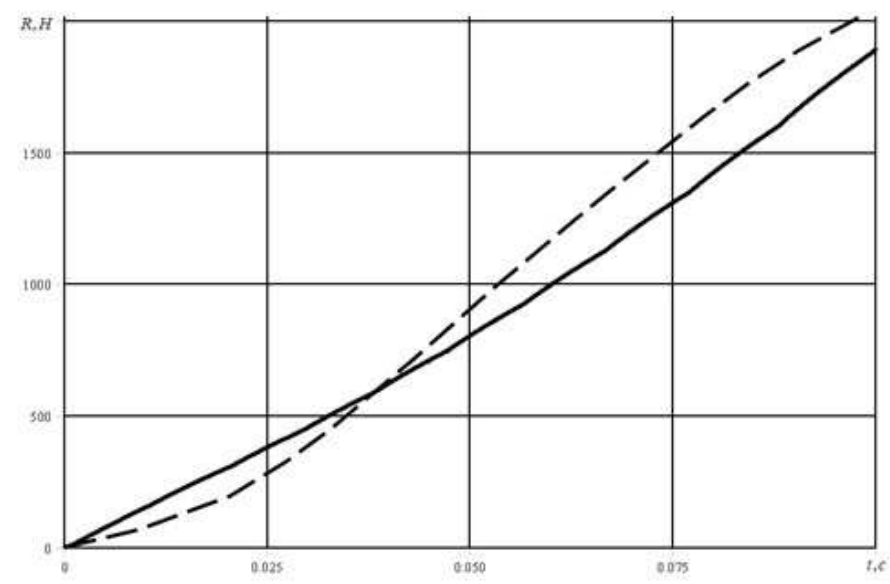

Figure 6: Contact pressure resultant curve.

\section{Calculation result}

As the example, we will show the results of calculation of unsteady contact interaction process for the sphere made of polyamide using the additive technologies. The shell and material parameters are described above. 
Figure 6 shows dependence of the resultant contact pressure $R$ upon time. Solid curve corresponds to theoretical result, dashed curve - experimental result.

\section{Acknowledgement}

The work was performed in Moscow Aviation Institute at financial support of the Federal goal-oriented program "Research and development of priority directions of Russia scientific-and-technological complex development on 2014-2020", Agreement No. 14.586.21.0019 (unique identificator RFMEFI58615X0019).

\section{References}

[1] E.Yu. Mikhailova, G.V. Fedotenkov, Nonstationary Axisymmetric Problem of the Impact of a Spherical Shell on an Elastic Half-Space (Initial Stage of Interaction), Mechanics of Solids, 46 (2011), 239-247. DOI: 10.3103/S0025654411020129

[2] D.V. Tarlakovskiy, G.V. Fedotenkov, Two-dimensional nonstationary contact of elastic cylindrical or spherical shells. Journal of Machinery Manufacture and Reliability, 43 (2014), 145-152. DOI: 10.3103/S1052618814010178

[3] D.V. Tarlakovskiy, G.V. Fedotenkov, Nonstationary 3D Motion of an Elastic Spherical Shell, Mechanics of Solids, 50 (2015), 208-217. DOI: 10.3103/S0025654415020107

[4] E.Yu. Mikhailova, D.V. Tarlakovskiy, G.V. Fedotenkov, Unsteady contact of spherical shell and elastic half-space, MAI Proceedings, 78 (2014), URL: http://www.mai.ru/upload/iblock/ 540/540b786eac60d751a2e5f5b8f745d731.pdf

[5] A.L. Medvedskiy, L.N.Rabinskiy, The method of surface features influence in nonstationary diffraction problems, MAI Press, Moscow (2007).

[6] S.I. Zhavoronok, M.Yu. Kuprikov, A.L. Medvedskiy, L.N. Rabinskiy, Numerical and analytical methods for solving problems of acoustic waves diffraction on a completely solids and shells, FIZMATLIT, Moscow (2010). 